\title{
Dispersion of the Prehistory Distribution: Analog Experiments and Numerical Results
}

\author{
M. Arrayás, ${ }^{1}$ J. M. Casado, ${ }^{2}$ J. Gómez Ordóñez, ${ }^{2}$ P. V.E. McClintock, ${ }^{1}$ M. Morillo,${ }^{2}$ and N. D. Stein ${ }^{1}$ \\ ${ }^{1}$ Department of Physics, Lancaster University, Lancaster, LA1 4YB, United Kingdom \\ ${ }^{2}$ Universidad de Sevilla, Física Teórica, Apartado Correos 1065, Sevilla 41080, Spain
}

(Received 11 November 1997)

\begin{abstract}
The dispersion parameter of the prehistory distribution for a potential system driven by white noise is analyzed theoretically and by means of analog and digital experiments. Nonmonotonic evolution of the dispersion with time is shown to arise provided that the potential fulfills a certain condition. It does not necessitate the existence of an unstable point, but can occur in single-minimum potentials, both symmetric and asymmetric. [S0031-9007(98)05610-5]
\end{abstract}

PACS numbers: 05.40. $+\mathrm{j}, 02.50 .-\mathrm{r}$

The macroscopic variables characterizing the behavior of very many physical systems are fluctuating quantities, and the fluctuations often become more pronounced as the system size decreases. The description of their time dependent probability laws is a formidable problem for which no general solution exists. Sometimes, one is interested mainly in stationary situations such that the one-time probability distribution has reached a time independent functional form. In such cases, it is well known that the small amplitude equilibrium fluctuations of stochastic variables around their stationary points are described by Gaussian distributions whose widths scale with the noise strength (which, in the case of thermal systems, corresponds to the temperature). In practice, however, it is often the large excursions of the system variables away from their steady values that are of greatest physical significance. These large rare fluctuations are responsible for many important physical phenomena including nucleation at phase transitions, switching between stable states in optically bistable systems, stochastic resonance, and the dynamics of Brownian ratchets. They are also of crucial importance in chemical kinetics where, for example, the reaction rate in thermal charge transfer reactions is largely determined by the statistics of large solvent fluctuations.

The statistical description of large rare fluctuations is a daunting problem [1-3]. One technique that has been used extensively to describe them relies on a path-integral formulation of the probability distribution (see, e.g., [4]). The (quasi)stationary probability density for large deviations from the average can be evaluated in terms of the optimal path originating from the neighborhood of the stationary point. A few years ago, a new approach to the investigation of large fluctuations was proposed, based on the idea of the prehistory distribution [5]. Briefly, this distribution can be defined as follows. Let us consider a system at equilibrium so that its one-time probability density is given by $P_{1}(x)$. As a consequence of a large fluctuation away from its steady value $x_{\mathrm{st}}$, the stochastic variable is observed to reach, for the first time at time $t_{f}$, its final value $x_{f}$. We are interested in the probability density $p_{h}\left(x, t ; x_{f}, t_{f}\right)$ that the system passed through a point $x$, intermediate between $x_{\mathrm{st}}$ and $x_{f}$, at an earlier time $t<t_{f}$. We will always consider that either $x_{\mathrm{st}}<x<x_{f}$ or $x_{\mathrm{st}}>x>x_{f}$. Where several stable points exist, we will be interested in time intervals much smaller than typical transition times between stable points, so that the termination point $x_{f}$ always lies within the same region of attraction as $x$ and $x_{\mathrm{st}}$.

By comparison of the results of analog electronic experiments with a path-integral calculation of the prehistory distribution, Dykman et al. showed [5] that, in the limit of very small noise strength, the time evolution of the maximum of the prehistory distribution follows the theoretically predicted optimal path. Indeed, following the maximum of the prehistory distribution is a convenient way of visualizing the optimal path in a real physical system where $D \neq 0$. There is a tube of trajectories around the optimal path whose width corresponds to the dispersion of the prehistory distribution. It was noted [5] that, for an overdamped particle moving in a bistable potential and subject to additive white noise, the dispersion could sometimes exhibit a nonmonotonic evolution with time: this unexpected feature was investigated both analytically and through the analog experiments. Subsequent numerical simulations corroborated these ideas [6] and allowed verification of the analytic results in the limit of very small noise intensity.

Dispersion of the prehistory distribution is important, not only for its own intrinsic interest, but also because it provides a direct measure of how useful the widely used (see, e.g., [2-4,7]) concept of the optimal path is going to be under the given circumstances: small dispersion implies that almost all paths lie close to the (idealized, $D \rightarrow 0$ ) optimal path, and vice versa.

The aim of this Letter is to analyze the behavior of the dispersion and, in particular, to clarify the relationship between its monotonicity and the shape of the potential in which the Brownian particle is moving. The work was prompted in part by the observation in [6] that the dispersion parameter shows a monotonic evolution with time along the optimal path when the termination point in a bistable potential is located farther from the local maximum of the potential than one of the stable points. Thus, 
the fact that the potential is bistable does not necessarily imply that the dispersion will evolve in a nonmonotonic fashion. Let us consider a simple system described by a variable $x(t)$ satisfying the Langevin equation (in dimensionless form)

$$
\dot{x}(t)=-U^{\prime}(x)+\xi(t),
$$

where $U(x)$ represents a potential and $\xi(t)$ is a Gaussian white noise with

$$
\langle\xi(t)\rangle=0, \quad\langle\xi(t) \xi(s)\rangle=D \delta(t-s) .
$$

In the limit of very small noise strength, the prehistory distribution is of Gaussian form, centered around the optimal path,

$$
\begin{aligned}
p_{h}\left(x, t ; x_{f}, t_{f}\right)= & {\left[2 \pi D \sigma\left(t ; x_{f}\right)\right]^{-1 / 2} } \\
& \times \exp \left(-\frac{\left[x-x_{\mathrm{opt}}\left(t ; x_{f}\right)\right]^{2}}{2 D \sigma\left(t ; x_{f}\right)}\right),
\end{aligned}
$$

where the optimal path, $x_{\text {opt }}\left(t ; x_{f}\right)$, is given as the solution of

$$
\dot{x}_{\text {opt }}(t)=U^{\prime}\left(x_{\text {opt }}\right) .
$$

The width of the Gaussian has been expressed as $D \sigma\left(t ; x_{f}\right)$ so that the dispersion parameter, $\sigma\left(t ; x_{f}\right)$, is independent of the noise strength. It is given by the expression [5]

$$
\begin{aligned}
\sigma\left(x ; x_{f}\right) & =\left[U^{\prime}(x)\right]^{2} \int_{x}^{x_{f}} d y\left[U^{\prime}(y)\right]^{-3}, \\
x & =x_{\mathrm{opt}}\left(t ; x_{f}\right) .
\end{aligned}
$$

Notice that $U^{\prime}(y) \neq 0$ for any point inside the integration interval.

For bistable potentials, the dispersion parameter as a function of $x$ can exhibit a nonmonotonic evolution when $x_{\mathrm{st}}<x<x_{f}<x_{\mathrm{un}}$ where $x_{\mathrm{un}}$ is the unstable point and $x_{\mathrm{st}}$ is one of the stable points [5]. This effect becomes more pronounced as the final point $x_{f}$ gets closer to the instability point. On the other hand, the behavior of $\sigma\left(x ; x_{f}\right)$ is monotonic when $x_{f}<x<x_{\text {st }}<x_{\text {un }}$ [6]. Clearly, the same holds true if one analyzes fluctuations around the other stationary point but with the signs of the inequalities reversed. For quartic potentials with a single minimum, $\sigma\left(x ; x_{f}\right)$ is monotonic regardless of whether the interval of interest is on the right or the left of the minimum. The question naturally arises, therefore, as to whether or not bistability is a necessary condition for the occurrence of nonmonotonic behavior of the dispersion parameter. We now point out that this is not, in fact, the case: the existence of nonmonotonic behavior is independent of the bistable/ monostable character of the potential. This can be seen by considering Eq. (5). Nonmonotonicity of the dispersion occurs if the slope of $\sigma\left(x ; x_{f}\right)$ is equal to zero at some point within the interval of interest. Equivalently, if for a given final point $x_{f}$ there exists at least one solution of the equation

$$
2 U^{\prime \prime}(x) \sigma\left(x ; x_{f}\right)-1=0
$$

in the interval $\left(x_{\mathrm{st}}, x_{f}\right)$, the dispersion will show a nonmonotonic behavior. Here $\sigma\left(x ; x_{f}\right)$ is given by Eq. (5). If (6) has several solutions, then $\sigma\left(x ; x_{f}\right)$ will show several extrema. If, for a given potential and final termination point $x_{f}$, no solution of (6) exists, then the dispersion parameter $\sigma\left(x ; x_{f}\right)$ will be monotonic.

To check these ideas we have carried out experiments and numerical simulations of the Langevin equation for several types of potential. Analog electronic circuits driven by white noise have been constructed [8] to model the equations of interest. Starting with the system in the vicinity of one stable state, successive blocks of $x(t)$ time series were digitized with a Microstar DAP3200A/ 415 ADC (analog-to-digital converter) [9], and examined. The moment at which $x(t)$ eventually reached a given final point $x_{f}$ was recorded, and the path followed by the system in reaching that point was also recorded. The process was then repeated, so as to build up an ensemble average of the paths leading to $x_{f}$. We have also solved the Langevin equation (1) numerically, using standard techniques [10]: the prehistory distribution and its parameters were obtained through similar procedures to those used in the analog experiments, but with an important difference. As discussed previously, provided that the detailed balance condition holds in the system under study, it can be exploited to evaluate the prehistory distribution in a relatively rapid and efficient manner [6]. For a Markov process with detailed balance, we can write

$$
p_{h}\left(x, t ; x_{f}, t_{f}\right)=w_{1 \mid 1}\left(x,\left|t-t_{f}\right| ; x_{f}\right), \quad\left(t<t_{f}\right),
$$

which shows that, for times $t$ prior to the final observation time $t_{f}$, the prehistory distribution can be found from a knowledge of the forward conditional probability density. Rather than waiting for the fluctuating system to arrive naturally at $x_{f}$ as in the analog experiments, therefore, we proceed by generating trajectories starting from $x_{f}$ at time $t_{f}=0$, and we allow the system to evolve forward in time. The construction of $p_{h}\left(x, t ; x_{f}, t_{f}\right)$ from the resultant stochastic trajectories is then straightforward. Note that this technique would not be applicable to systems for which detailed balance does not hold. This is the case in the model analyzed by Maier and Stein [3]. Even though an equilibrium distribution exists, the potential conditions are not satisfied for certain values of the parameters characterizing the model, and the system lacks detailed balance. The technique is also inadequate for externally driven systems where a time independent equilibrium distribution does not exist. These last two examples, showing that the timereversal symmetry of the fluctuations is broken, have been recently studied in [11]. 
As a first explicit example, let us consider the potential

$$
U(x)=-\frac{x^{2}}{2}+\frac{x^{4}}{20}-20 x,
$$

which is asymmetric around the single minimum located at $x_{\mathrm{st}}=5$. A numerical analysis of Eq. (6) indicates that it has a single solution when $x_{f}<x_{\mathrm{st}}$, while no solution exists for $x_{f}>x_{\mathrm{st}}$. Thus, one expects that the prehistory distribution will have a width with a nonmonotonic dependence on time in the first case, while its behavior should be monotonic when $x_{f}>x_{\mathrm{st}}$. For instance, for $x_{f}=2.49$, Eq. (6) has a single root at the point $x=4$.14. In Fig. 1, we plot the results of the analog experiments and the numerical solution of the Langevin equation for two different values of $x_{f}$ located on either side of the potential minimum. Agreement between the results obtained through these very different approaches may be regarded as satisfactory. The analog data reach the abscissa axis slightly farther from the potential minimum than the chosen $x_{f}$ in each case on account of the coarse graining in coordinate and time introduced by the ADC, which means that there is on average some overshoot before a crossing of $x_{f}$ is detected, and the same effect also causes the maximum value of $\sigma$ to be underestimated. The numerical trajectories, by contrast, start from the chosen value of $x_{f}$ in each case (prior to the use of time reversal under the detailed balance condition, as discussed above), so there is no possibility of an overshoot. The most important feature, clearly shown by both the analog and numerical results, is the marked difference in behavior according to whether $x_{f}$ is larger or smaller than $x_{\mathrm{st}}$.

When the final point is to the right of the minimum, $x_{f}=7.0>x_{\mathrm{st}}$, the dispersion is monotonic, just as ex-

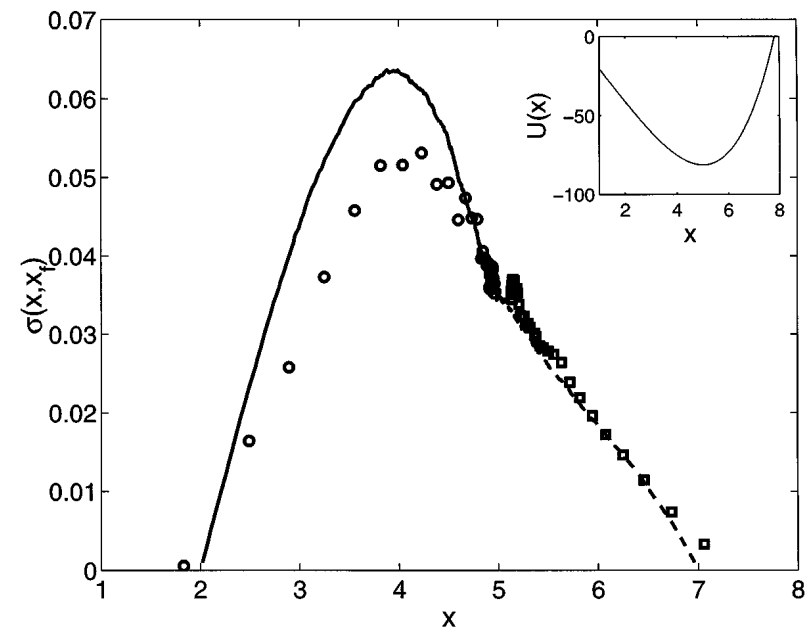

FIG. 1. Dispersion parameter for the asymmetric singleminimum potential (8) plotted in the inset. Numerical (curves) and analog experimental (points) data with $D=4$ are shown for arrivals at two different termination points: $x_{f}=2.0$ (circles and full curve) and $x_{f}=7.0$ (squares and dashed curve), respectively. At large negative times, the system approaches its stationary state at $x=5$ in both cases. pected. When $x_{f}=2.0<x_{\mathrm{st}}$, on the other hand, there is a clear nonmonotonicity of $\sigma\left(x ; x_{f}\right)$. Its maximum occurs very near the theoretically predicted position. Thus, nonmonotonic behavior can indeed exist even in the absence of an instability point in the potential. It should be mentioned that the matching of the experimental and numerical results provides further [11] corroboration of the expectation that, when detailed balance holds, the main fluctuational paths away from the stable point are time-reversed mirror images of the relaxational paths towards it.

As a second example, we consider the prehistory problem for a more complicated potential that has been used to describe several aspects of the dynamics of fluctuations in SQUIDS (superconducting quantum interference devices) [12]

$$
U(x)=0.15(x+2 \pi)^{2}-\cos (x) .
$$

This potential has a single minimum at $x_{\mathrm{st}}=-2 \pi$, about which it is symmetric, as seen in the inset of Fig. 2. The existence of solutions of Eq. (6) depends upon the location of $x_{f}$. For $x_{f}$ very close to $x_{\mathrm{st}}$, there are no solutions of Eq. (6), and, therefore, $\sigma\left(x ; x_{f}\right)$ is monotonic. Actually, it follows from Eq. (5) that, regardless of the shape of the potential, the dispersion is always monotonic provided that $x_{f}$ is sufficiently close to the stable point. Once the final point is sufficiently far from the stable point, however, solutions of Eq. (6) exist. Their number increases as the distance of the observation point from the minimum is increased. A new extremum of the dispersion parameter appears corresponding to each of these solutions. In Fig. 2, we compare the results of the numerical simulations and the analog experiments for the potential of Eq. (9), for a noise strength $D=2.54$ and $x_{f}=3$. This end point is far from the minimum of the potential, and Eq. (6)

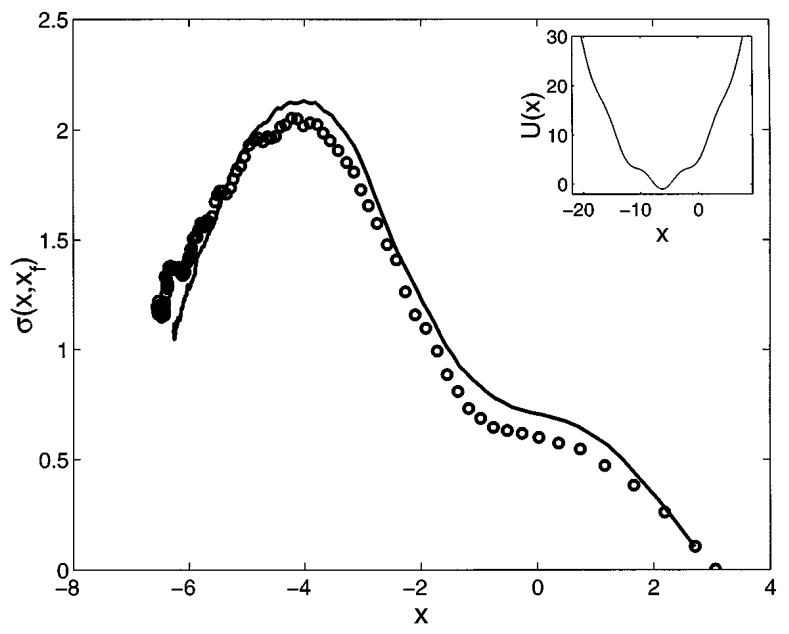

FIG. 2. Dispersion parameter for the symmetric singleminimum potential (9) plotted in the inset. Numerical (curve) and analog experimental (points) data with $D=2.54$ are shown for arrivals at $x_{f}=3$. At large negative times, the system approaches its stationary state at $x=-2 \pi$. Only one extremum is resolved. 
has three roots located at $x_{1}=0.89, x_{2}=-0.52$, and $x_{3}=-4.41$. It is clear from the plots that the dispersion parameter shows a very distinct maximum located at $x \approx$ -4 , but that the other two predicted extrema do not appear. The shift in the position of the main maximum and the absence of the other two extrema are both attributable to the finite value of $D$ used in the experiments. Note that Eq. (6) was derived in the asymptotic $D \rightarrow 0$ limit, so that it cannot necessarily be expected to give good results for finite noise values. To test this explanation, we have repeated the numerical experiments for much weaker noise intensity, with $D=10^{-8}$. The results are presented in Fig. 3 in the form of a semilog plot to exhibit the extrema to best advantage. The numerical error can be estimated from the technique used (fourth-order RungeKutta with 1000 trajectories, a time step of 0.001 , and a total run time of 15000 ), from the total number of trajectories or, perhaps more conveniently, directly from the small statistical deviations that are visible about a smooth curve in the figure. In all cases, one concludes that the numerical uncertainty is considerably smaller than the difference $(0.137)$ between the $\sigma\left(x, x_{f}\right)$ values computed at the local minimum and the smaller local maximum. It is clear, therefore, that for this very small value of $D$ all three extrema are unambiguously resolved.

In conclusion, analog experimental and numerical solutions of Langevin equations show that the dispersion parameter of the prehistory distribution as a function of position along the optimal path can have a nonmonotonic dependence on $x=x_{\mathrm{opt}}$, even for monostable, symmetric potentials. The monotonicity property is related to the global aspects of the potential indicated by Eq. (6). The theoretical predictions are asymptotically valid in the limit of very small noise intensity. Experimental data are always obtained for finite noise strength, and their comparison with theoretical predictions requires finite noise effects to be properly taken into account. Even for finite noise, the comparison between the numerical and experimental data can be regarded as very satisfactory. On the other hand, stochastic simulations based on the detailed balance property can be carried out for extremely small values of $D$. Thus, they provide results which are well within the asymptotic limit where they can be matched directly against theoretical predictions.

We acknowledge valuable discussions with M. I. Dykman and D. G. Luchinsky. This work was supported by British Council/Spanish Acciones Integradas Exchange Program, by the Junta de Andalucía, by the Dirección General de Investigación Científica y Técnica of Spain under Project No. PB95-0536, by the EC DG-XII, by

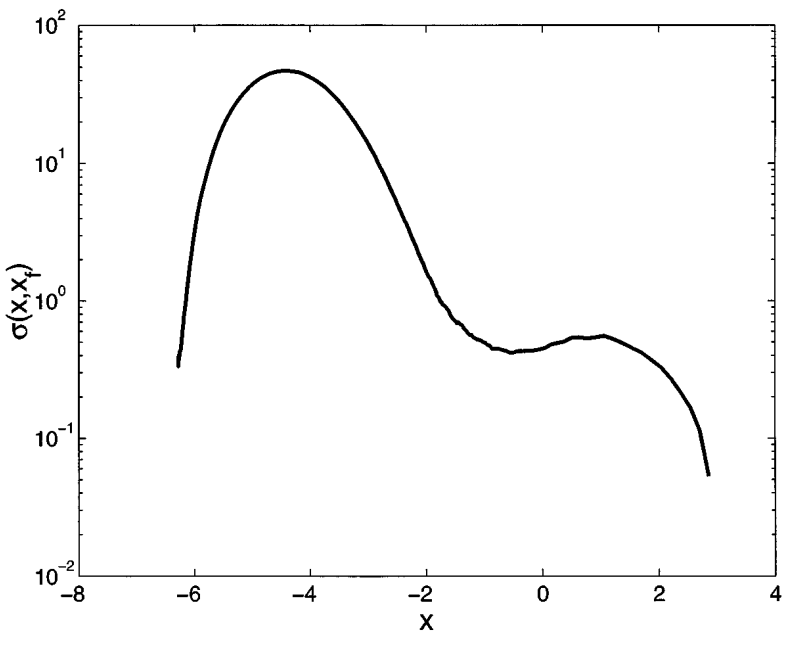

FIG. 3. Semilogarithmic plot of the dispersion parameter for the same symmetric single-minimum potential of Fig. 2 for $x_{f}=3$, but with $D=10^{-8}$. The results were obtained by numerical solution of the Langevin equation. Three extrema are clearly resolved.

INTAS, and by the Engineering and Physical Sciences Research Council (U.K.).

[1] D. Ludwig, SIAM Rev. 17, 605 (1975).

[2] M. Dykman and K. Lindenberg, in Contemporary Problems in Statistical Physics, edited by G. H. Weiss (SIAM, Philadelphia, 1994), pp. 41-101.

[3] R.S. Maier and D. L. Stein, Phys. Rev. Lett. 71, 1783 (1993).

[4] A. J. McKane, H. C. Luckock, and A. J. Bray, Phys. Rev. A 41, 644 (1990); A. J. Bray, A. J. McKane, and T. J. Newman, Phys. Rev. A 41, 657 (1990).

[5] M. I. Dykman, P. V.E. McClintock, V.N. Smelyanski, N. D. Stein, and N. G. Stocks, Phys. Rev. Lett. 68, 2718 (1992).

[6] J. Gómez Ordóñez, J. M. Casado, and M. Morillo, Phys. Rev. E 54, 2125 (1996); M. Morillo, J. M. Casado, and J. Gómez Ordóñez, Phys. Rev. E 55, 1521 (1997).

[7] M. I. Dykman, E. Mori, J. Ross, and P. M. Hunt, J. Chem. Phys. 100, 5737 (1994).

[8] L. Fronzoni, in Noise in Nonlinear Dynamical Systems, edited by P. V.E. McClintock and F. Moss (Cambridge University Press, Cambridge, 1989), Vol. 3, pp. 222-242.

[9] Microstar Laboratories Inc., 2265 116th Avenue NE, Bellevue, WA 98004.

[10] See, for instance, H.S. Greenside and E. Helfand, Bell Syst. Tech. J. 60, 1927 (1981).

[11] D. G. Luchinsky and P. V.E. McClintock, Nature (London) 389, 463 (1997).

[12] R. L. Kautz, Rep. Prog. Phys. 59, 935 (1996). 